\title{
Experience of Excess Skin and Attitude to Body Contouring Surgery of a Chinese Post-Bariatric Population
}

\author{
Zhiyuan Jiang $^{\mathrm{a}}$ Guixiang Zhang $^{\mathrm{a}}$ Xiao $\mathrm{Du}^{\mathrm{a}, \mathrm{b}}$ Yi Chen $^{\mathrm{a}}$ Chaoyong Shen $^{\mathrm{a}}$ \\ Zhen Cai $^{\mathrm{c}}$ Bo Zhang ${ }^{\mathrm{a}}$ Zhong Cheng ${ }^{\mathrm{a}}$ \\ aDepartment of Gastrointestinal Surgery, West China Hospital, Sichuan University, Chengdu, China; \\ bDepartment of General Surgery, Ya'an People's Hospital, Ya'an, China; 'Department of Plastic Surgery, Sichuan \\ Provincial People's Hospital, University of Electronic Science and Technology of China, Chengdu, China
}

\section{Keywords}

Post-bariatric · Excess skin · Body contouring surgery ·

Chinese patients $\cdot$ Cross-sectional study

\begin{abstract}
Introduction: Western studies have explored post-bariatric patients concerning their views on excess skin and body contouring surgery (BCS), but Asian data were lacking. This study aims to investigate the experience of excess skin and attitude to BCS of a Chinese post-bariatric population. Methods: A total of 210 Chinese patients who underwent bariatric surgery from March 2015 to September 2018 were cross-sectional studied using the Sahlgrenska Excess Skin Questionnaire and a study-specific questionnaire. Results: The survey response rate was $61.4 \%$. Most responders (78.2\%) reported they had excess skin, and the most common sites were the abdomen (70.2\%) and the upper arms (61.3\%). Most responders $(66.1 \%)$ reported being bothered by impaired health-related quality of life (HRQoL), and the most common problem was "the feeling of having unattractive body appearance" (42.7\%). Many patients (37.9\%) desired for BCS, and "the impact of excess skin is not serious enough" was the reason why not undergoing BCS being chosen most (28.1\%),
\end{abstract}

then "the cost is too high" (20.2\%) and "worrying about the risk or complications of BCS" (18.4\%). Younger age, female gender, higher weight loss, having full-time job, and earning higher income were independent factors increasing their desires for BCS. Conclusions: Most Chinese post-bariatric patients have excess skin and are bothered by impaired HRQoL. The abdomen and upper arms are the sites where patients are most seriously affected and most eager for BCS. The conservative attitude toward BCS and the cost without reimbursement are the main barriers.

(c) 2021 The Author(s)

Published by S. Karger AG, Basel

\section{Introduction}

Obesity (body mass index $[\mathrm{BMI}] \geq 30 \mathrm{~kg} / \mathrm{m}^{2}$ ) is one of the greatest public health issues in many countries, and its prevalence is increasing rapidly worldwide in recent decades [1]. Obesity increases the risk of comorbidities such as diabetes mellitus, hypertension, hyperlipidemia, obstructive sleep apnea, and cardiovascular diseases that

Zhiyuan Jiang and Guixiang Zhang contributed equally to the research.
C 2021 The Author(s).

Published by S. Karger AG, Basel

This is an Open Access article licensed under the Creative Commons Attribution-NonCommercial-4.0 International License (CC BY-NC) (http://www.karger.com/Services/OpenAccessLicense), applicable to the online version of the article only. Usage and distribution for commercial purposes requires written permission.
Correspondence to:

Bo Zhang, hxwcwk@126.com

Zhong Cheng, zhongcheng63@126.com 
all affect human health and reduce life expectancy $[2,3]$. According to the Endocrine Society Scientific Statement on the Science of Obesity Management, programs that are effective for weight loss include peer-reviewed and approved lifestyle modification programs, diets, commercial weight loss programs, exercise, medications, and surgery [4].By changing the anatomy and physiology of the gastrointestinal tract, bariatric/metabolic surgeries such as sleeve gastrectomy and Roux-en-Y gastric bypass impact appetite, eating behavior, metabolism, energy expenditure, and other mechanisms to produce substantial weight loss and improve obesity-related comorbidities [2, $3,5,6]$. In comparison with Caucasians with the same BMI, Asians have a higher percentage of body fat and visceral fat, which has predisposed Asians to insulin resistance and the development of type 2 diabetes and associated metabolic disorders at lower BMI levels [7]. As a result, a need for bariatric surgery is becoming apparent for Asian patients with obesity [8].

Although weight loss following bariatric surgery is almost beneficial, it also involves adverse effects; the main downside is the excess skin which could develop in many body parts [9]. It is not uncommon for post-bariatric patients to report a high level of dissatisfaction with excess skin which leads to social withdrawal, physical discomfort, psychological issues and, eventually, impairments to health-related quality of life (HRQoL) [10-12]. The problems related to excess skin cannot be completely resolved by modifications in lifestyle, or physical exercise [13], while body contouring surgery (BCS) which refers to a series of plastic procedures aiming to eliminate excess skin and adipose tissues seems to be the only solution to be effective $[14,15]$. According to the latest available global statistics from the International Society of Aesthetic Plastic Surgery, surgical procedures involving the body and extremities, such as abdominoplasty, buttock lift, and lower body lift, increased by $7 \%$ within just 1 year, reaching almost 3.5 million surgeries performed annually [16].

Quite a few clinical studies focused on the post-bariatric excess skin, some assessed predictors of BCS utilization in bariatric patients and reported the prevalence of BCS, and some evaluated the effect of BCS on excess skinrelated symptoms, and some described the desire for as well as barriers to BCS [16-21]. Also, several systematic reviews and meta-analyses confirmed the positive effects of BCS on HRQoL and concluded that most post-bariatric patients desired $\mathrm{BCS}[13,22,23]$. However, all these studies were carried out in Western developed countries and taken the Caucasian race as the main object, thus lacked data from developing countries (running different health insurance policies) and Mongolians (having different skin characteristics). Collectively, the present crosssectional study was designed to investigate the experience of excess skin of Chinese post-bariatric population and their desire for as well as barriers to BCS.

\section{Materials and Methods}

\section{Study Design and Patients}

This is a cross-sectional study among the patients of our prospective database. The database starts on March 2015 and enrolls patients who underwent bariatric surgery at the department of Gastrointestinal Surgery of West China hospital. The inclusion criteria of patients in this study are as follows: (1) Mongolians with Chinese nationality; (2) undergoing bariatric surgery before September 2018; (3) no experience of massive weight loss (MWL) [24] before bariatric surgery; (4) contact can be made through WeChat; and (5) not suffering from any malignant tumor or disabling disease.

A total of 210 patients were identified eligibly and then were sent a questionnaire survey in October 2020 via WeChat-based electronic questionnaire platform (Wenjuanxing, http://www.wjx. $\mathrm{cn} /)$. Before starting the questionnaire, patients gave electronic informed consent. Patients were asked to fill in their real name, age, preoperative weight and approximate time of bariatric surgery in the questionnaires so that we could make sure that the questionnaires were written by themselves, and only when these basic information of a questionnaire matches with that in the database, it is regarded as an effective response. The patients who did not reply were sent the same questionnaire a second time 2 weeks later. Each patient can submit the questionnaire only once, and the submitters with effective responses were considered as responders. Variables including nationality, race, gender, degree of education, follow-up, baseline body height, baseline body weight, type of bariatric procedure, comorbidities with obesity, and complications of bariatric surgery were collected from the database.

\section{Questionnaires}

Chinese version Sahlgrenska Excess Skin Questionnaire (SESQ)

Following the format and structure of its original version, we translated the SESQ into Chinese for the first time. The Chinese version SESQ also contains 29 questions divided into 3 parts: Part 1 contains several questions concerning demographic data; Part 2 includes assessment of symptoms caused by excess skin (HRQoL); and Part 3 includes questions about the amount and degree of discomfort from excess skin on specific parts of the body.

\section{Study-Specific Questionnaire}

The study-specific questionnaire was constructed in order to: (1) obtain more detailed general information such as educational level, employment, complications of bariatric surgery, and chronic diseases and (2) determine the prevalence of BCS, desires for BCS, and the reasons not undergoing BCS of our post-bariatric patients.

\section{Statistical Analyses}

Descriptive statistics are given as mean and SD, as median and range, or as number and percentage. Continuous variables in the different groups were tested for normal distribution using the Kruskal-Wallis normality test. The independent-sample $t$ and the 
Table 1. Basic characteristics of study population

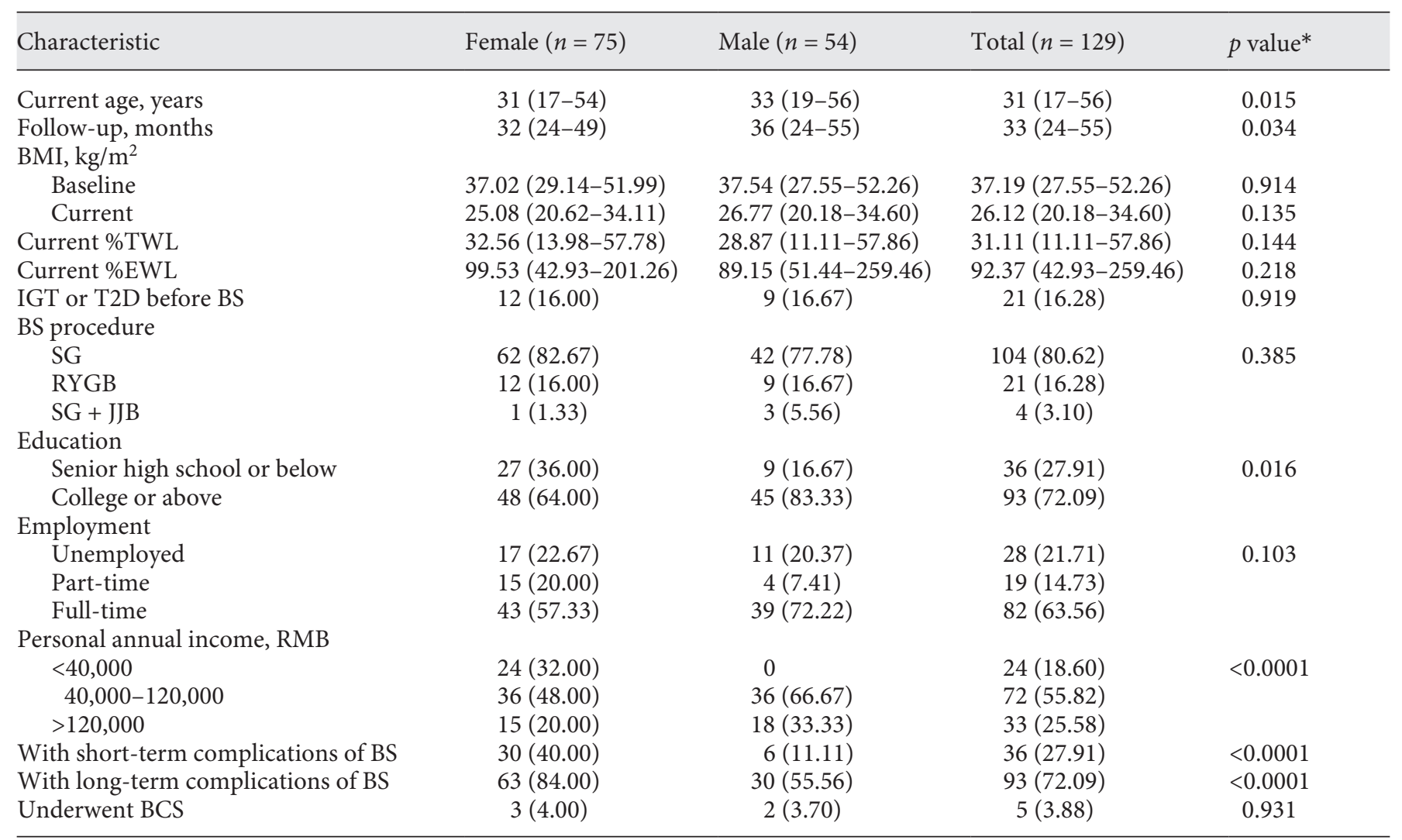

Values are given as median (range) or $n$ (\%). BMI, body mass index; \%TWL, percent of total body weight loss; \%EWL, percent of excess weight loss; IGT, impaired glucose tolerance; T2D, type 2 diabetes; BS, bariatric surgery; SG, sleeve gastrectomy; GB, gastric bypass; JJB, jejunojejunal bypass; BCS, body contouring surgery; RYGB, Roux-en-Y gastric bypass. * Group comparison according to gender using either Mann-Whitney U or Pearson $\chi^{2}$ tests.

Mann-Whitney U tests were used for the data subject to normal distribution and abnormal distribution, respectively, for comparisons between different groups. Categorical variables were assessed using the Pearson $\chi^{2}$ or the Fisher exact test, as appropriate. The pairwise correlation among the percent of total body weight loss (\%TWL), the amount of excess skin, and the impairments to HRQoL were analyzed using Spearman's rank correlation coefficient. Multivariable logistic regression analysis was performed to determine independent factors associated with desire for BCS. $p$ value $\leq 0.05$ (2-sided) was considered to indicate a statistically significant difference. All statistical analysis was performed with SPSS 25 statistical software (IBM Corporation, Armonk, NY, USA).

\section{Results}

\section{Basic Characteristics of Study Population}

Of 210 eligible patients, 129 (61.43\%) responded effectively and were counted as study population. The major- ity of the study population were adults and female, and only 4 were adolescent at the time of bariatric surgery. Most of them received higher education, $54(41.86 \%)$ had bachelor's degrees, and 12 (9.30\%) got master's or doctoral degrees. Most of them (78.29\%) were employed, and $96(74.42 \%)$ patients earned 120,000 RMB or less. Only 3 (2.33\%) patients did not reach MWL after surgery with $\%$ EWL being $42.93,44.92$, and $43.86 \%$, respectively. Of the study population, only 3 females and 2 males underwent a total of 8 BCS procedures, including 4 performed in the abdomen, 3 in the upper arms and 1 in the chin. According to the grouping of female and male, there was no statistical difference in baseline BMI, current BMI, pre-bariatric impaired glucose tolerance or type 2 diabetes, type of bariatric surgery, employment, and prevalence of BCS. The details of basic characteristics are listed in Table 1. 


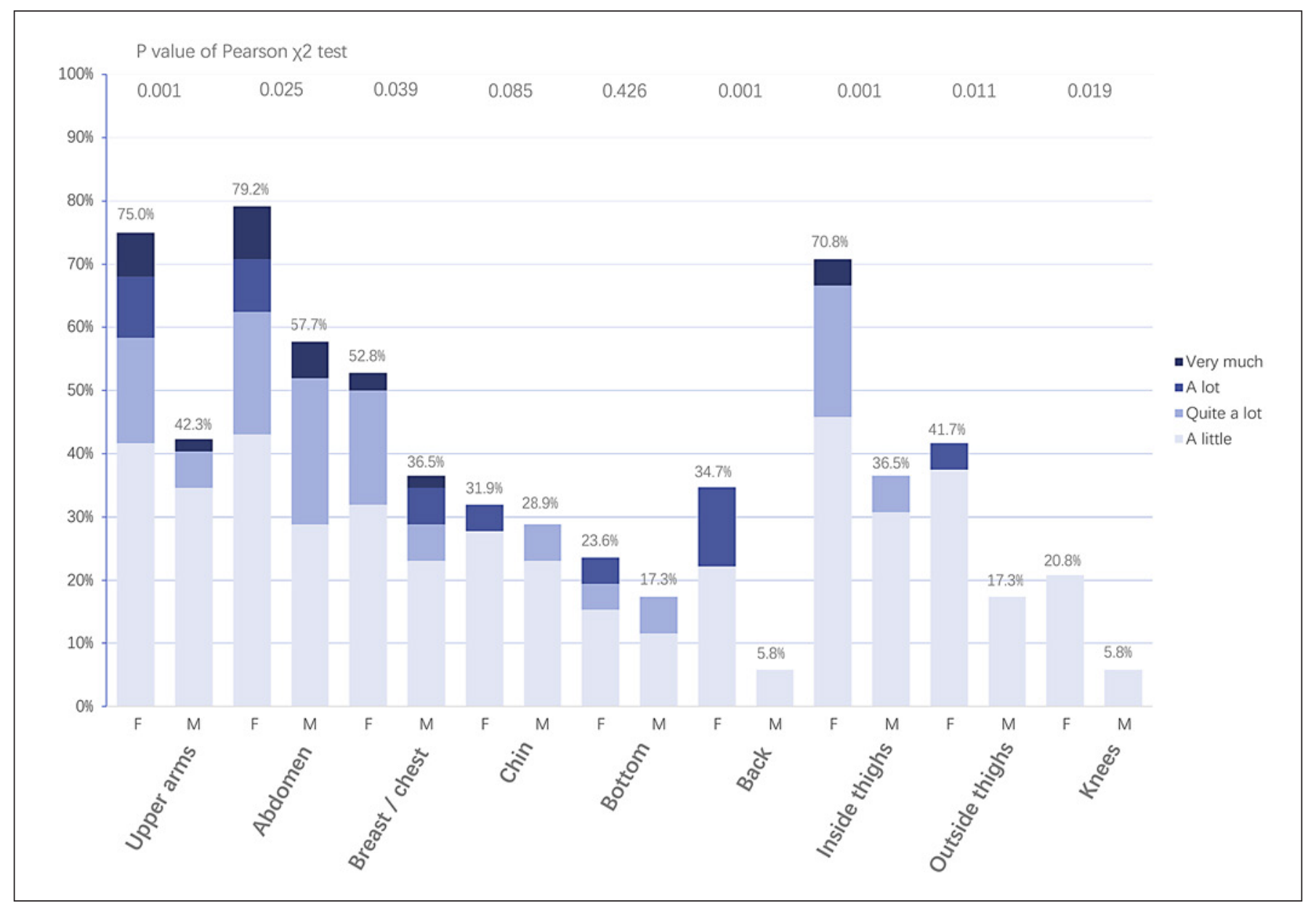

Fig. 1. Assessment of the amount of excess skin on different body parts.

\section{Experience of Excess Skin}

The experiences of excess skin of 124 patients who had not received any post-bariatric BCS were studied; results are shown in Figure 1 and online suppl. Table 1; see www. karger.com/doi/10.1159/000517587 for all online suppl. material. Most of responders $(83.33 \%$ of females and $71.15 \%$ of males) reported they had excess skin. Females expressed higher proportions of having excess skin than males, with statistically significant differences $(p<0.05)$ in most sites except for the chin and buttocks. The most frequently reported sites of having excess skin in both genders were the abdomen $(79.17 \%$ in female and $57.69 \%$ in male), followed by the upper arms (75.00\% in female and $42.31 \%$ in male). For the responses that estimated the amount of excess skin as "very much" or "a lot," the abdomen was the most common site in females (16.67\%) and busts the most in males (7.69\%).
The findings concerning the impairments to HRQoL due to excess skin are shown in Figure 2 and online suppl. Table $2.66 .67 \%$ of females and $65.38 \%$ of males reported being bothered by impaired HRQoL. The most common problem was the feeling of having unattractive body appearance $(47.22 \%$ in female and $36.54 \%$ in male). The males reported higher proportions in "pain in upper back" and "hindrance in intimate situations" than females ( $p=0.031$ and 0.036 , respectively), while female patients had more "difficulties to find clothes that fit" $(p=0.026)$. No statistical difference was found in the remaining 7 examined dimensions between men and women $(p>0.05)$.

\section{Attitude to BCS}

Of the 124 patients who had not undergone any BCS procedure, 47 (37.90\%) expressed a desire for BCS, and in detail, $57.45 \%$ of them rated their desires as "occasional- 


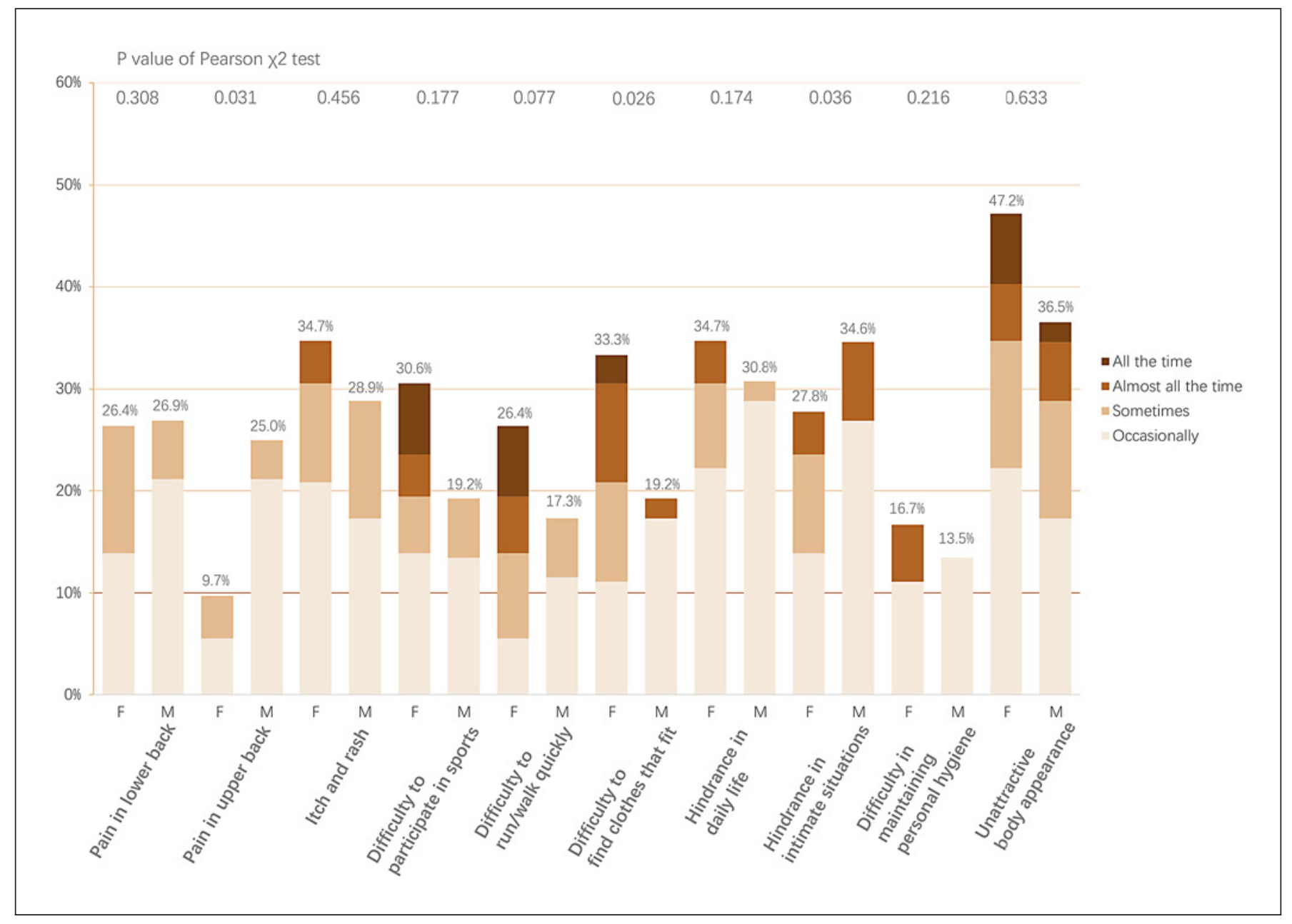

Fig. 2. Impairments because of excess skin to health-related quality of life.

ly," $36.17 \%$ as "sometimes," and $6.38 \%$ as "all the time." Multivariable analyses suggested that younger age, female gender, higher \%TWL, having full-time job and earning higher annual income were independent factors of patients' desires for BCS (Table 2). Thirty-eight patients responded the question of "the top 3 body parts you want most to underwent BCS" and reported 87 body parts, of which the "abdomen" was chosen 32 times (36.78\%), followed by the "upper arms" 18 times (20.69\%), the "thighs" and "breasts/chest" both 17 times (19.54\%), and finally the "waist" 3 times (3.45\%). At the first blank of the top 3 body parts, 14 patients chose the "abdomen," 12 the "breasts/chest," 9 the "upper arms," and 3 the "thighs."

Sixty-four patients reported a total of 114 reasons why not undergoing BCS (Fig. 3). "The amount of excess skin or its impact on HRQoL is not serious enough to require BCS” was the reason chosen for the most, up to 32 times.
"Thinking that the cost is too high" and "worrying about the risk, complications or surgical scars brought by BCS" were 2 common reasons chosen by more than 20 times, respectively. More than 10 patients either thought that they needed more information before making a decision or planned to undergo BCS in the near future.

\section{Discussion}

To the best of our knowledge, this study is the first one to investigate the experience of excess skin and attitude to BCS of Asian post-bariatric patients. Previous studies suggested that a major weight loss occurred within the first year after bariatric surgery $[25,26]$, and BCS was often deferred until 18 months to ensure weight stability [27], thus we enrolled patients who had underwent bar- 


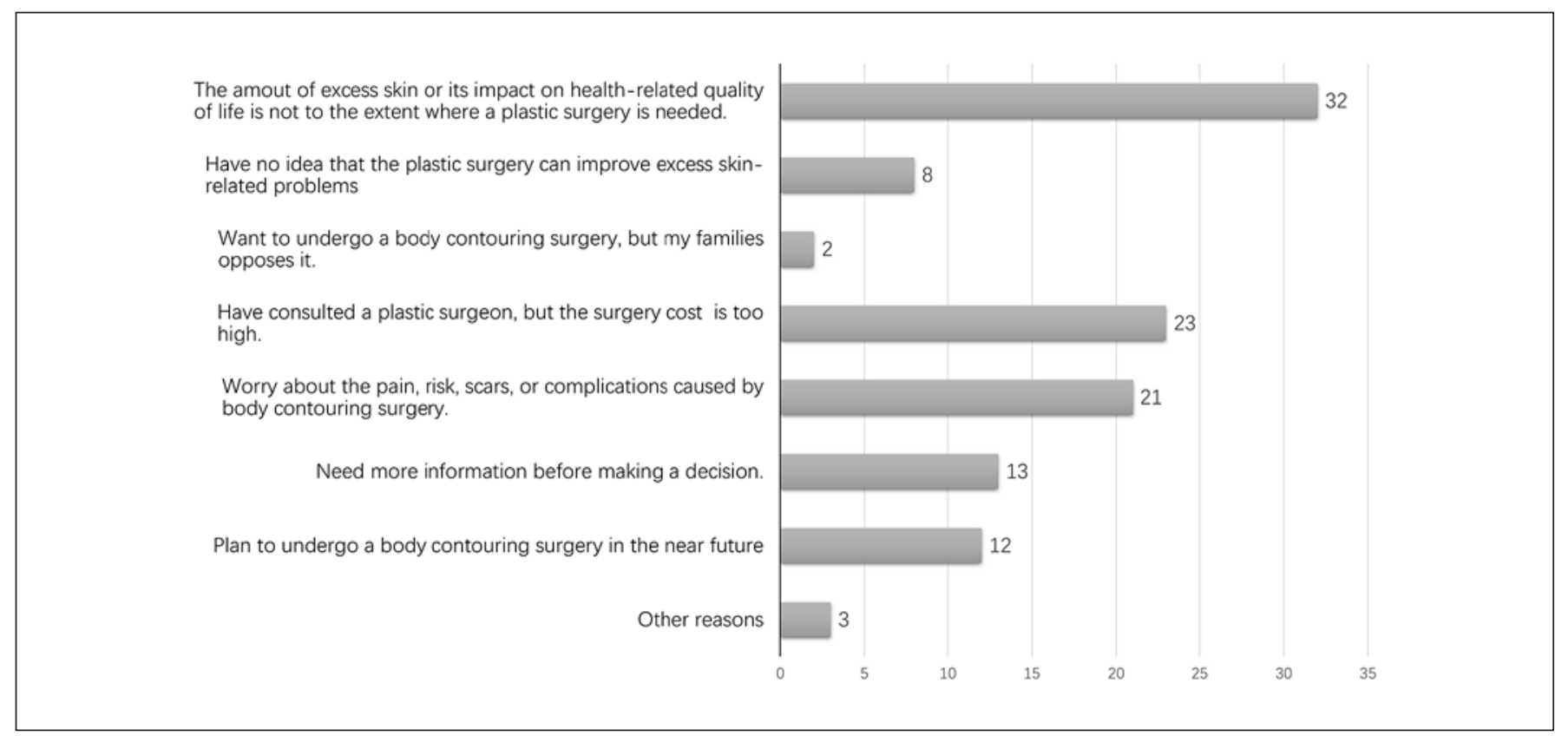

Fig. 3. Reasons why not undergoing body contouring surgery.

Table 2. Multivariable logistic analyses of covariates associated with desire for BCS

\begin{tabular}{|c|c|c|c|}
\hline & OR & $95 \% \mathrm{CI}$ & $p$ value \\
\hline Age & 0.866 & $0.784-0.957$ & 0.005 \\
\hline \multicolumn{4}{|l|}{ Gender } \\
\hline Male & Ref & & \\
\hline Female & 7.789 & $1.646-36.853$ & 0.010 \\
\hline$\%$ TWL & 1.109 & $1.040-1.183$ & 0.002 \\
\hline$\%$ EWL & 0.996 & $0.973-1.019$ & 0.708 \\
\hline \multicolumn{4}{|l|}{ Short-term complication } \\
\hline Without & Ref & & \\
\hline With & 0.606 & $0.108-3.410$ & 0.570 \\
\hline \multicolumn{4}{|l|}{ Long-term complication } \\
\hline Without & Ref & & \\
\hline With & 1.100 & $0.252-4.777$ & 0.899 \\
\hline \multicolumn{4}{|l|}{ Education } \\
\hline Senior high school or below & Ref & & \\
\hline College or above & 0.407 & $0.076-2.184$ & 0.294 \\
\hline \multicolumn{4}{|l|}{ Employment } \\
\hline Unemployed & Ref & & \\
\hline Part-time & 0.415 & $0.047-3.694$ & 0.431 \\
\hline Full-time & 8.072 & $1.403-46.441$ & 0.019 \\
\hline \multicolumn{4}{|l|}{ Personal annual income, RMB } \\
\hline$<4,000$ & Ref & & \\
\hline $4,000-12,000$ & 7.462 & $1.208-46.106$ & 0.031 \\
\hline$>12,000$ & 12.861 & $1.299-127.327$ & 0.029 \\
\hline The amount of excess skin & 1.156 & $0.972-1.375$ & 0.101 \\
\hline The impairments to HRQoL & 1.150 & $0.973-1.359$ & 0.101 \\
\hline
\end{tabular}

BS, bariatric surgery; GB, gastric bypass; JJB, jejunojejunal bypass; \%TWL, percent of total body weight loss; \%EWL, percent of excess weight loss; HRQoL, health-related quality of life; BCS, body contouring surgery. 
iatric surgery more than 24 months in the hope that the body weight of the most and the troubles caused by excess skin had stabilized, and the attitudes toward BCS surgery had been mature.

There had been no specific instrument in Chinese for evaluating post-bariatric patients' experience of excess skin. After browsing a number of quality-of-life measurement instruments for bariatric and BCS, we finally chose the SESQ which was regarded as the most suitable one by all team members and translated it into Chinese. SESQ is the first questionnaire to evaluate excess skin from the patient's point of view and its test-retest reliability as well as validity have been proved to be good [28].

E-mail is not popular among Chinese ordinary people, a considerable number of them do not have an e-mail address, and the proportion of them who regularly use it to deal with personal affairs is very low. Therefore, WeChat known as the social software most used by Chinese people was chosen for our study. We imported the content of our questionnaire into a network platform (Wenjuanxing, http://www.wjx.cn/), through which a QR code link associated with this questionnaire was established. The QR code can be shared through WeChat, and patients who receive it are able to fill out the questionnaire immediately. Before releasing the $\mathrm{QR}$ code, we first evaluated the convenience of the whole process of answering the questionnaire, and then invited several patients to evaluate its readability and understandability. Then, we made several improvements and distributed the final version to all eligible patients. Within 2 weeks, we received 91 valid responses, and after sending a reminder, we received another 38 ones, thus adding up to 129 responders, with a response rate of $61.43 \%$ which was about the same as that of similar Western studies that surveyed patients via email [29].

The World Health Organization identified for Asian populations potential public health action points of BMI $\geq 23 \mathrm{~kg} / \mathrm{m}^{2}$ (increased risk) and BMI $\geq 27.5 \mathrm{~kg} / \mathrm{m}^{2}$ (high risk) [30]. The median of \%EWL of this study population was $92.37 \%$, and $97.67 \%$ of them reached MWL, which not only showed the well effect of bariatric procedures to these people, but also affirmed the characteristics of their body weights from the side, that is, they were mainly with class I and II obesity. After all, because of the peculiarity of obesity in Asians (higher percentage of visceral adipose tissue) and their vulnerability to insulin resistance [31], they may consider receiving surgical intervention when with lower BMI than western counterparts.

To rule out the effect of BCS, we excluded 5 patients who had undergone BCS before surveys when analyzing

Chinese Patients' Experience of Excess Skin experiences of excess skin. The results showed that like Western studies, most Chinese patients developed more or less excess skin in some area of the body, and reported being bothered by impaired HRQoL. Generally, females were more likely to develop excess skin, and the prevalence of excess skin in the abdomen, upper arms, inner thighs, and breasts/chest were all more than half. For the responses that estimated the amount of excess skin as "very much" or "a lot," the abdomen was also the most common site reported to have the most severe degree of excess skin, which agreed basically with the results of other studies $[10,28]$. The proportion of patients suffered from the impairments to HRQoL was not as high as that of feeling excess skin, even the most common symptom caused by excess skin "unattractive body appearance" occurred in less than half in both sexes. Even if the proportions were not equal, a strong correlation $\left(r_{\mathrm{s}}=0.668, p<\right.$ 0.0001 ) was found between the amount of excess skin and the impairments to HRQoL, indicating that the excess skin is an important factor in determining the HRQoL; details can be found in online suppl. Table 3 .

The urgency of obtaining BCS was not as intense as in Western studies with $<40 \%$ patients expressing expectations for BCS. As for the reasons why not undergoing a BCS procedure, unlike Western patients that often listed "cost" or "lack of reimbursement" in the first place, "the impact of excess skin is not serious enough to require BCS" was chosen by the half of 64 responders. The above 2 points revealed these patients' more conservative attitude toward BCS. In addition, 23 responders said they gave up BCS because of the high costs; Western developed countries could reimbursed patients' part or all of the BCS expenses [15, 17, 32], but the Chinese basic medical insurance and all commercial insurances both list BCS as a purely cosmetic surgery and refuse coverage, which further reduces the desire for BCS and prevalence of BCS. At last, there were quite a few patients who had no idea concerning whether BCS could alleviate excess skin-related problems, and worried too much about the scars or surgical risks caused by BCS, which likely suggests that both bariatric and plastic surgeons should correctly promote BCS in clinical practice.

Previous works have shown that women, those of younger ages, those with a greater change in BMI, and those with poorer psychosocial self-report scores were associated with desiring BCS and pursuit of BCS after bariatric surgery $[9,12]$. Not quite similar to their results, multivariable analysis in the current investigation revealed that variables were identified as independent factors to increase patients' desires for BCS including young- 
er age, female gender, and higher \% TWL as well as having full-time job and earning higher personal annual income. The reason why the last 2 variables are included may be related to the fact that all expenses of a BCS procedure could be paid only out of patient's own pocket in China, thus the patients with full-time jobs and higher incomes have more initiative in deciding whether or not to undergo BCS.

Though the present study adopted strict inclusion criteria for eligible patients and valid responders, conducted with rigorous statistical analysis, and obtained the firsthand information concerning the experiences of excess skin of Asians patients, it has some limitations. First, this study was limited by cross-sectional design, which provided the evidence not at the highest quality. Second, the survey was administered only once at patients' 2 to 4 -year postoperative time points, so the impact of heterogeneity of follow-up time on the results cannot be ruled out, studies have pointed out that patients' attitudes towards excess skin and BCS changed over time [27]. Third, we used self-translated and self-made questionnaires and did not physically examine or measure the patients, therefore, we could obtain no raw data that can be matched and compared with that of other researches. The fourth point was the lack of data from nonresponders, patients with greater problems with excess skin are more likely to response to this survey than those with less problems, thus we are at a risk of over-interpretation of the results. At last, the proportion of individuals who underwent BCS was very small so that we could not explore the differences about excess skin and HRQoL that may exist between those who did and did not undergo BCS.

\section{Conclusion}

Most Chinese post-bariatric patients especially females have excess skin in one or more body parts, and many of them are bothered by impaired HRQoL due to excess skin, which can be seen in aspects of physical symptoms as well psychosocial function. However, the desires for BCS are not as intense as reported in Western studies, and attitudes toward BCS are more conservative. Also, the higher cost lacking any reimbursement is another important barrier to obtaining BCS. The abdomen and upper arms are the sites where Chinese patients are most likely to have excess skin, are most seriously affected by it, and are most eager for BCS as well as are the sites where the most BCS procedures have been performed. Younger age, female gender, higher weight loss, having full-time job, and earning higher personal annual income were independent factors increasing patients' desires for BCS. Few studies have focused on excess skin issues of Asian post-bariatric patients, and more research attention is needed here.

\section{Statement of Ethics}

This research protocol was approved by West China Hospital of Sichuan University Biomedical Research Ethics Committee (No. 2020137). All procedures performed in this study involving human participants were in accordance with the 1964 Declaration of Helsinki and its later amendments. Only patients who view and confirm the electronic informed consent on the home page of the questionnaire can continue to complete it, so all the valid questionnaires are in line with the principle of informed consent.

\section{Conflict of Interest Statement}

The authors have no conflicts of interest to declare.

\section{Funding Sources}

This study was supported by the Wu Jieping Medical Foundation (320.2710.1810) and 1.3.5 project for disciplines of excellence, West China Hospital, Sichuan University (ZYJC18034).

\section{Author Contributions}

The original idea of this research was conceived by Z. Jiang, B. Zhang, and Z. Cheng. Z. Jiang and G. Zhang recruited patients, distributed the questionnaires, collected and analyzed data, and drafted the manuscript. X. Du., Y. Chen., C. Shen., and Z. Cai. were actively involved in questionnaire translation and design, statisti$\mathrm{cal}$ analysis, data interpretion, and contributed to the discussion. B. Zhang and Z. Cheng supervised the work and had full access to all the data in this study and takes responsibility for the integrity as well as the accuracy of the data. All the authors approved the final version of the manuscript.

\section{References}

1 Nam GE, Kim YH, Han K, Jung JH, Rhee EJ, Lee SS, et al. Obesity fact sheet in Korea, 2019: prevalence of obesity and abdominal obesity from 2009 to 2018 and social factors. Jomes. 2020;29(2):124-32.

2 Ashrafian H, Harling L, Toma T, Athanasiou C, Nikiteas N, Efthimiou E, et al. Type 1 diabetes mellitus and bariatric surgery: a systematic review and meta-analysis. Obes Surg. 2016;26(8):1697-704.

3 Hussain A. The effect of metabolic surgery on type 1 diabetes: meta-analysis. Arch Endocrinol Metab. 2018 Mar-Apr;62(2):172-8. 
4 Bray GA, Heisel WE, Afshin A, Jensen MD, Dietz WH, Long $\mathrm{M}$, et al. The science of obesity management: an endocrine society scientific statement. Endocr Rev. 2018;39(2):79132.

5 Pucci A, Batterham RL. Mechanisms underlying the weight loss effects of RYGB and SG: similar, yet different. J Endocrinol Invest. 2019;42(2):117-28.

6 Al-Najim W, Docherty NG, le Roux CW. Food intake and eating behavior after bariatric surgery. Physiol Rev. 2018;98(3):1113-41.

7 Yoon KH, Lee JH, Kim JW, Cho JH, Choi YH, $\mathrm{Ko} \mathrm{SH}$, et al. Epidemic obesity and type 2 diabetes in Asia. Lancet. 2006;368(9548):1681-8

8 Seki Y, Kasama K, Kikkawa E, Yokoyama R, Nabekura T, Sano A, et al. Five-year outcomes of laparoscopic sleeve gastrectomy in Japanese patients with class I obesity. Obes Surg. 2020;30(11):4366-74.

9 Marek RJ, Steffen KJ, Flum DR, Pomp A, Pories WJ, Rubin JP, et al. Psychosocial functioning and quality of life in patients with loose redundant skin 4 to 5 years after bariatric surgery. Surg Obes Relat Dis. 2018;14(11): 1740-7.

10 Kitzinger HB, Abayev S, Pittermann A, Karle B, Bohdjalian A, Langer FB, et al. After massive weight loss: patients' expectations of body contouring surgery. Obes Surg. 2012; 22(4):544-8.

11 Krauss S, Medesan R, Black J, Medved F, Schaefer R, Schaller HE, et al. Outcome of body-contouring procedures after massive weight loss. Obes Surg. 2019;29(6):1832-40.

12 Steffen KJ, Sarwer DB, Thompson JK, Mueller A, Baker AW, Mitchell JE. Predictors of satisfaction with excess skin and desire for body contouring after bariatric surgery. Surg Obes Relat Dis. 2012 JanFeb;8(1):92-7.

13 Toma T, Harling L, Athanasiou T, Darzi A, Ashrafian $\mathrm{H}$. Does body contouring after bariatric weight loss enhance quality of life? A systematic review of QOL studies. Obes Surg. 2018;28(10):3333-41.

14 Derderian SC, Dewberry LC, Patten L, Sitzman TJ, Kaizer AM, Jenkins TM, et al. Excess skin problems among adolescents after bariatric surgery. Surg Obes Relat Dis. 2020; 16:9938.
15 Monpellier VM, Antoniou EE, Mulkens S, Janssen IMC, Jansen ATM, Mink van der Molen $A B$. Body contouring surgery after massive weight loss: excess skin, body satisfaction, and qualification for reimbursement in a dutch post-bariatric surgery population. Plast Reconstr Surg. 2019;143(5):1353-60.

16 Paul MA, Opyrchał J, Knakiewicz M, Jaremków P, Duda-Barcik Ł, Ibrahim AMS, et al. The long-term effect of body contouring procedures on the quality of life in morbidly obese patients after bariatric surgery. PLoS One. 2020;15(2):e0229138.

17 de Vries CEE, Kalff MC, van Praag EM, Florisson JMG, Ritt MJPF, van Veen RN, et al. The influence of body contouring surgery on weight control and comorbidities in patients after bariatric surgery. Obes Surg. 2020;30(3): 924-30.

18 Klassen AF, Kaur M, Breitkopf T, Thoma A, Cano S, Pusic A. Using the BODY-Q to understand impact of weight loss, excess skin, and the need for body contouring following bariatric surgery. Plast Reconstr Surg. 2018; 142(1):77-86.

19 Vierhapper MF, Pittermann A, Hacker S, Kitzinger HB. Patient satisfaction, body image, and quality of life after lower body lift: a prospective pre- and postoperative long-term survey. Surg Obes Relat Dis. 2017;13(5):8827.

20 Poulsen L, Klassen A, Rose M, Roessler KK, Juhl CB, Støving RK, et al. Patient-reported outcomes in weight loss and body contouring surgery: a cross-sectional analysis using the BODY-Q. Plast Reconstr Surg. 2017;140(3): 491-500.

21 Staalesen T, Olbers T, Dahlgren J, Fagevik Olsén $M$, Flodmark CE, Marcus C, et al. Development of excess skin and request for bodycontouring surgery in postbariatric adolescents. Plast Reconstr Surg. 2014;134(4): 627-36.

22 Ivezaj V, Grilo CM. The complexity of body image following bariatric surgery: a systematic review of the literature. Obesity Reviews. 2018;19(8):1116-40.
23 Gilmartin J, Bath-Hextall F, Maclean J, Stanton W, Soldin M. Quality of life among adults following bariatric and body contouring surgery: a systematic review. JBI Database System Rev Implement Rep. 2016;14(11):24070 .

24 Shermak MA, Chang D, Magnuson TH, Schweitzer MA. An outcomes analysis of patients undergoing body contouring surgery after massive weight loss. Plast Reconstr Surg. 2006;118(4):1026-31.

25 Courcoulas AP, Christian NJ, Belle SH, Berk PD, Flum DR, Garcia L, et al. Weight change and health outcomes at 3 years after bariatric surgery among individuals with severe obesity. JAMA. 2013;310(22):2416-25.

26 Inge $\mathrm{TH}$, Courcoulas AP, Jenkins TM, Michalsky MP, Helmrath MA, Brandt ML, et al. Weight loss and health status 3 years after bariatric surgery in adolescents. $\mathrm{N}$ Engl J Med. 2016;374(2):113-23.

27 Derderian SC, Patten L, Kaizer AM, Inge TH, Jenkins TM, Michalsky MP, et al. Body contouring in adolescents after bariatric surgery. Surg Obes Relat Dis. 2020;16(1):137-42.

28 Staalesen T, Fagevik Olsén M, Elander A. Experience of excess skin and desire for body contouring surgery in post-bariatric patients. Obes Surg. 2013;23(10):1632-44.

29 Jiang Z, Zhang G, Huang J, Shen C, Cai Z, Yin $\mathrm{X}$, et al. A systematic review of body contouring surgery in post-bariatric patients to determine its prevalence, effects on quality of life, desire, and barriers. Obes Rev. 2021;22: e13201.

30 WHO Expert Consultation. Appropriate body-mass index for Asian populations and its implications for policy and intervention strategies. Lancet. 2004;363(9403):15763.

31 Lear SA, Humphries KH, Kohli S, Chockalingam A, Frohlich JJ, Birmingham CL. Visceral adipose tissue accumulation differs according to ethnic background: results of the multicultural community health assessment trial (MCHAT). Am J Clin Nutr. 2007;86(2):353-9.

32 Azin A, Zhou C, Jackson T, Cassin S, Sockalingam S, Hawa R. Body contouring surgery after bariatric surgery: a study of cost as a barrier and impact on psychological well-being. Plast Reconstr Surg. 2014;133(6):776e-82e. 